\title{
Electron Interference in a Quantum Ring with a Tunable Magnetic Field Shield
}

\author{
Received 15 February, 2021; accepted 2 March, 2021
}

\author{
Minky Seo ${ }^{\mathrm{a}, \mathrm{b}}$, Hwanchul Jung ${ }^{\mathrm{b}}$, and Yunchul Chung ${ }^{\mathrm{b}, *}$ \\ a Division of Physical Metrology, Korea Research Institute of Standards and Science, Daejeon 34113, Republic of Korea \\ b Department of Physics, Pusan National University, Busan 46241, Republic of Korea
}

\author{
*Corresponding author E-mail: ycchung@pusan.ac.kr
}

\begin{abstract}
A quantum ring device that can turn on and off the external magnetic field on the ring is developed. The device is to study the effect of the interaction between electrons and external magnetic fields on Aharonov-Bohm interference. The switching is realized by employing a superconducting shield gate and turning on and off the superconductivity on the gate with applied current or magnetic field. The electron interference due to Aharonov-Bohm effect is observed regardless of the presence of the external magnetic field on the electron path of the ring. The result is consistent with the fact that effects of potentials on charged particles exists even in the region where all the field vanish hence no direct interaction between electron and magnetic field is allowed.
\end{abstract}

Keywords: Aharonov-Bohm effect, Nano ring, Electron interference, Superconductivity, GaAs/AlGaAs, Single electron transistor

\section{Introduction}

Electron interference observed by varying magnetic field in a quantum ring is the manifestation of Aharonov-Bohm (AB) effect [1]. The interference is the results of the phase difference between two electron beams (see Fig. 1(a), rotating clockwise and counter-clockwise directions) which is proportional to the enclosed magnetic flux inside a quantum ring. According to Aharonov and Bohm, it is not necessary for electron to experience the magnetic field directly. They explicitly mentioned that "contrary to the conclusions of classical mechanics, there exist effects of potentials on charged particles, even in the region where all the fields (and therefore the forces on the particles) vanish" [1]. In other words, the charged particle is affected by the vector potential even though the particle is confined in the region where the magnetic field is zero, which is totally surprising and the result of quantum mechanics. Many efforts have been followed to realize the experiment that Aharonov and Bohm proposed (in other words to isolate the electron path from the external magnetic field). By using toroidal ferromagnet covered with superconductor layer, Tonomura et al. observed electron holography produced by electron beams passing the toroid [2]. This experiment is recognized as the evidence and the first experimental realization of Aharonov and Bohm effect. Despite the successful demonstration of $A B$ effect, there were some skepticism and attempts to explain the effect under the framework of classical physics [3-5], including interaction between electron and magnetic fields.

In this work, we developed a quantum ring device that can turn on and off the external magnetic field on the electron path of the ring. The device enables us to measure the $\mathrm{AB}$ interference under two different situations, i) when electrons are directly exposed to an external magnetic field and ii) when the electrons are shielded from an external magnetic. The shielding is done by placing a superconducting gate on top of the electron path of the ring. Application of a small current on the gate destroyed the superconducting state of the gate and exposed electrons to an external magnetic field. The measurements were done at $50 \mathrm{mK}$. The results show almost no difference between two cases, the visibilities and the frequencies of the interferences were almost identical. The result reconfirms the original idea of Aharonov and Bohm and is against the recent idea that the interference is due to the classical interaction between electron and the external magnetic field.

\section{Experimental details}

Figures 1(a)-(c) are showing the concept of the device. A quantum ring is formed by applying negative voltages on the gates painted in green. The negative voltages on the green gates will stop electrons flow under the gate. Thus, the electron will flow only along the trajectories denoted as red arrows in Figs. 1(a) and 1(b) [6-8]. To switch on and off the magnetic field on the electron path, while allowing magnetic flux pass through the area enclosed by the electron path, a superconducting gate (the gate colored in blue) is placed to cover only the electron path, as shown in Figs. 1(c) and 1(d). Note that the ring of the superconducting shield gate is not forming a closed circle in Fig. 1(d). This is to avoid the flux quantization inside the superconducting ring, which hinders the continuous change of magnetic field in the ring. A precise alignment is necessary to avoid short circuits between ordinary gates forming quantum ring and superconducting shield gate. A small misalignment (less than $50 \mathrm{~nm}$ ) is observed in Fig. 1 (d) but without any visible short between gates. The red circular gate in Fig. 1(d) is connected via so called a "bridge gate" (see Fig. 1(e) for a 3-dimensional view) because a direct connection to the outer region is blocked by the superconducting ring gate. Finally, four gray gates on either side of the ring tunes the coupling between the reservoir and the ring.

The quantum ring is fabricated on a 2-dimensional electron gas (2DEG) wafer based on a GaAs/AlGaAs heterostructure with electron density of $3 \times 10^{11} \mathrm{~cm}^{-2}$ and mobility of $2.1 \times 10^{6} \mathrm{~cm}^{2} \mathrm{~V}^{-1} \mathrm{~s}^{-1}$ at $4.2 \mathrm{~K}$. The $2 \mathrm{DEG}$ is buried $62 \mathrm{~nm}$ below the surface of the wafer. Prior to the 

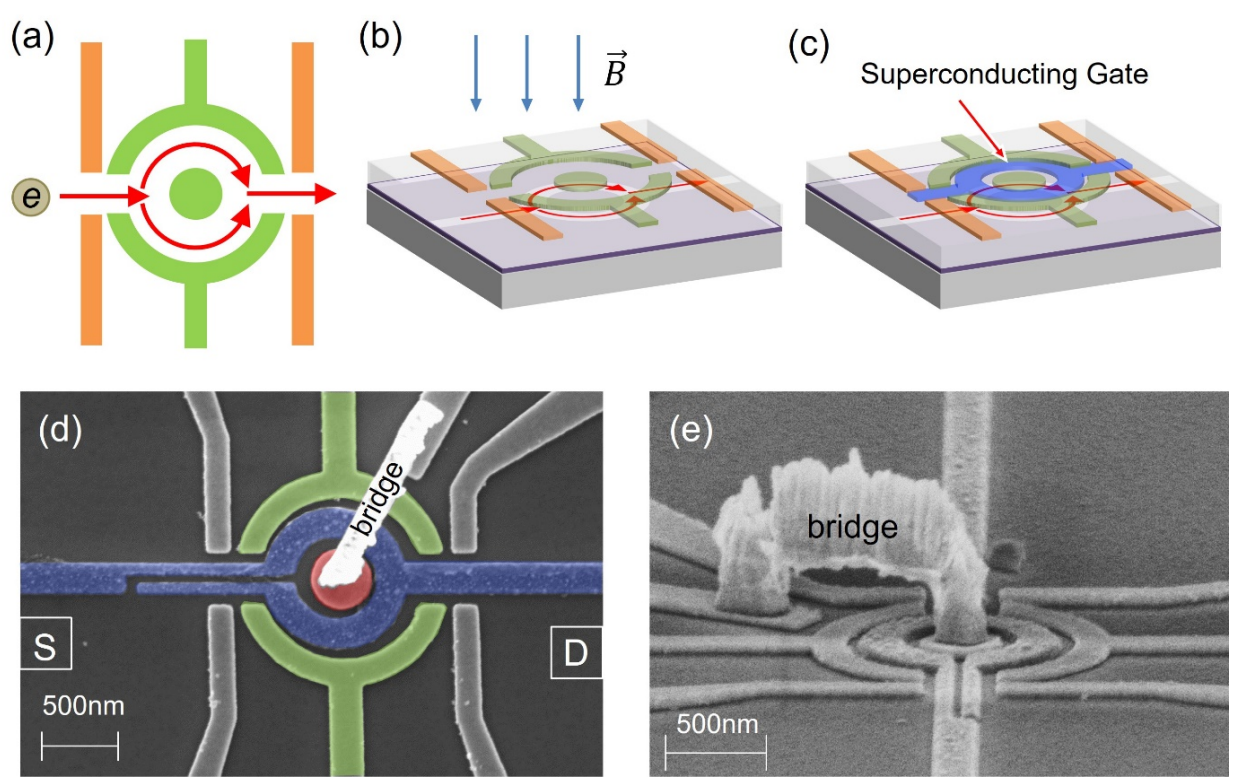

Figure 1. Figures showing the concept and SEM pictures of the device. (a) A quantum ring without external magnetic field shield on the electron path. (b) The same figure viewed from the side. (c) A device that blocks an external magnetic field on the electron path using a superconducting gate (blue gate). (d) SEM picture of the device. Green and red gates are defining a quantum ring while green gates on either side of the ring tunes the coupling between quantum ring and the electron reservoir. The source and drain of the device are denoted as ' $S$ ' and ' $D$ ', respectively. The white gate noted as a "bridge" connects the inner red circular gate to the outer region of the device. (e) The side view of a bridge gate.
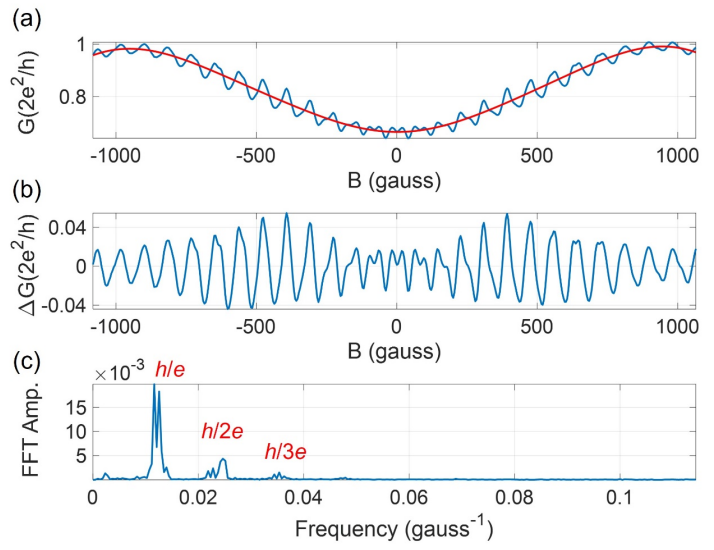

Figure 2. (a) The conductance through the device measured as a function of external magnetic fields (blue trace). The AB oscillation was observed over a slowly varying background (red trace) as a function of the magnetic fields. To extract only the $\mathrm{AB}$ oscillation data, a smooth background (red trace) is subtracted from the measured data (blue trace). (b) The result after subtraction. (c) The frequencies of the $A B$ oscillations obtained by the fast Fourier transformation (FFT) of the measured data.

gate fabrication, mesa etching and ohmic contact evaporation/annealing are done $[9,10]$. Three gate evaporations are done to complete the device fabrication. First, the quantum ring and coupling gates (green, red and gray gates in Fig. 1(d)) are defined by using electron beam lithography and 25/25 nm-thick Ti/Au layers are evaporated. Second, a shield gate is fabricated with $50 \mathrm{~nm}$ thick $\mathrm{Al}$ layer (which is superconducting up to around $1 \mathrm{~K}$ in temperature) by using same method mentioned above. Finally, a bridge gate is fabricated by electron beam lithography on a PMMA/MAA 8.5 MAA bi-layer resistor [11]. All the measurements are done at $50 \mathrm{mK}$ in a dilution refrigerator. The conductance through the device is measured by applying an excitation voltage of $10 \mu \mathrm{V}$ from the source and measuring the current on the drain. The measurements were done as a function of external magnetic fields and the ring gate (green gates in Fig. 1(a)) voltages.

\section{Results and discussion}

Figure 2(a) shows typical magnetoconductance data measured for a fixed ring gate voltage. Fast oscillation of conductance as a function of magnetic field, in addition to a relatively slowly varying conductance background, is observed. The oscillation period matches with a flux quanta $h / e$, which is the fingerprint of $\mathrm{AB}$ oscillations [6-8]. A smooth background (red trace) is subtracted from the raw data to extract conductance changes related to the $\mathrm{AB}$ oscillations only, which is shown in Fig. 2(b). A smooth background is obtained by fitting the data with a polynomial function which uses sinusoidal functions as bases. The result shows the $\mathrm{AB}$ oscillation having a periodicity of around 83 gauss (corresponds to the one flux quanta of the ring). Also, an extra oscillation with a shorter periodicity is evident. Fast Fourier transformation result in Fig. 2(c) shows the oscillations with integer multiples of the fundamental $\mathrm{AB}$ frequency. Such high frequency oscillations exist due to the electrons rotating multiple times in the ring $[6,8]$. Regardless of their frequencies, the oscillations are still the direct consequence of $A B$ effect. Note that the observed $\mathrm{AB}$ oscillation shows mirror symmetry around zero magnetic field. In other words, $G(B)=G(-B)$, where $G$ is the conductance of the device and $B$ is the external magnetic field. This is due to the Onsager relation based on time-reversal symmetry of two-terminal closed-ring device, which was reported by Yacoby et al. [7].

Figure 3(a) shows the AB oscillation (after subtracting smooth background) measured by varying external magnetic fields and the voltages on the ring gate (green gate in Fig. 1(d)). The AB oscillations are observed in the entire range of the magnetic field. It is important to notice that the magnetic field shielding above a critical field is not available due to the breakdown of superconductivity in the gate. Figure 3 (b) shows the shield gate resistance as a function of magnetic fields. From the magnetoresistance curve, it is clear that the electron is not shielded (from the external magnetic fields) outside the region shaded in gray (roughly between \pm 350 gauss). The small increase in resistance at the magnetic fields around \pm 250 gauss (denoted by arrows in the figure) is due to the early breakdown of superconductivity in the narrower strip (painted in red) of the gate. The reason for splitting a gate into two parts (painted in red) is to make an open ring to prevent 

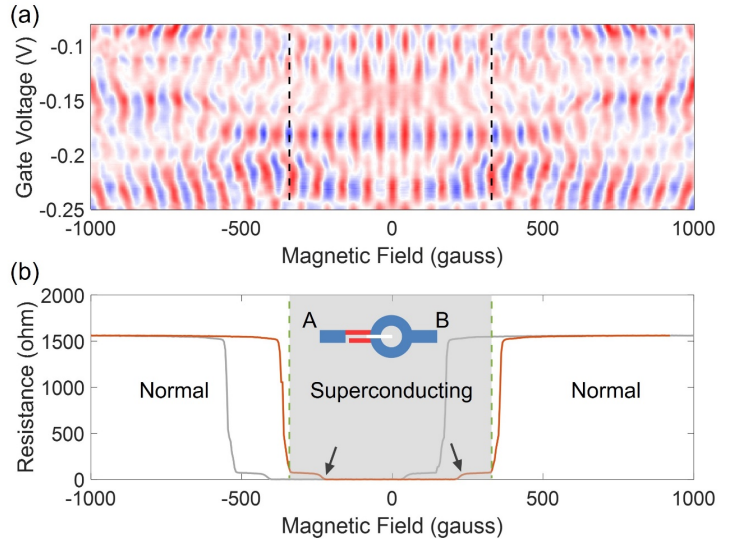

Figure 3. (a) The AB oscillation (after subtracting smooth background) observed by varying external magnetic fields and gate voltages on the ring. (b) The resistance of a superconducting gate (measured between A and B) as a function of external magnetic fields. Inset shows the shape of the superconducting shield gate. The red (gray) trace is measured while ramping up (down) the magnetic fields. There was a noticeable hysteresis due to the remanent magnetization of the magnet and its surroundings. Hence, (a) is measured while ramping up the field.
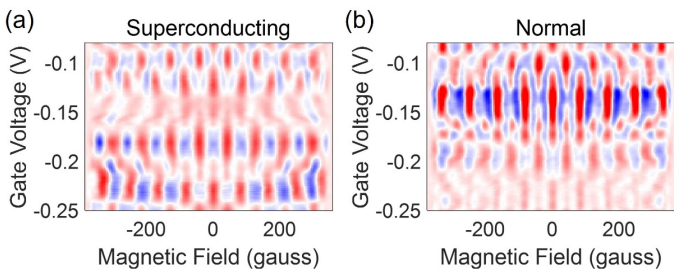

Figure 4. $A B$ oscillations measure when the shield gate is in (a) a superconducting state and (b) a normal state. To break the superconductivity on the shield gate a small current $(2.9 \mu \mathrm{A})$, which is above the critical current of the shield gate, is applied.

flux quantization $[12,13]$ in the ring. Despite the breakdown of superconductivity in a narrower strip of the gate, the ring gate still maintains superconducting state. Hence the electron path is still shielded until the magnetic field exceeds \pm 350 gauss in amplitude.

If the origin of the $\mathrm{AB}$ interference is due to the classical interaction between electrons and the magnetic fields, it is not expected to observe any interference in the region where the external field is shielded (between black dashed lines in Fig. 3(a)). However, AB interference is clearly present in the region. Also, note that there is no abrupt change of the interference patterns at the interfaces (black dashed line) between shielding and non-shielding region. This observation also supports that $\mathrm{AB}$ interference does not require direct interaction between electron and magnetic fields.

Another way of suppressing the superconductivity on the gate is to apply a current higher than the critical current of the gate. The measured critical current (measured by applying current thought the gate and monitoring the resistance of the gate) of our shield gate was around $0.8 \mu \mathrm{A}$, which is about an order of magnitude smaller than the reported value with a similar structure [14]. We believe that the discrepancy is due to the differences in quality and geometry (thickness and width) of the Aluminum gates. Due to the discrepancy, we applied a current of $2.9 \mu \mathrm{A}$, which is well above the measured critical current, to suppress the superconductivity on the gate. Figures $4(\mathrm{a})$ and 4 (b) are the $A B$ interferences measured without and with the current on the gate, respectively. Again, AB interferences are observed regardless of shielding. Even though, the two figures are not identical, overall features such as visibility and the interference patterns are pretty much the same for both cases (superconducting vs normal). The visibilities, which is defined as $\left(G_{\max }-G_{\min }\right) /\left(G_{\max }+G_{\min }\right)$, of the interference were around $0.03-0.05$ for both cases. Also, the 2-dimensional interference patterns look similar even though the visibilities, at specific gate voltages, are slightly different. The discrepancy in the visibility is due to the slow changes in the gate characteristics (the conductance as a function gate voltage), which is quite common for GaAs/AlGaAs heterostructure based devices. Since it takes about 12 hours for each measurement, a slight change in the gate characteristic is expected, which results in somewhat different graphs shown in Fig. 4. Considering that the interferences are observable for both cases (current on and off), despite of small differences, we believe the results also support that $\mathrm{AB}$ interference does not require direct interaction between electron and magnetic fields.

\section{Conclusions}

A quantum ring which can turn on and off the external magnetic field on the electron path of the ring is developed. The switching is realized by employing a superconducting shield gate and controlling the superconductivity on the gate with applied current or magnetic field. The device enables us to test if the direct interaction between electron and the magnetic field is necessary to observe $A B$ interference. Our results show that the $A B$ interference is hardly affected by the existence of direct interaction between the electron and the magnetic field. The result reconfirms the fact that "the effects of potentials on charged particles exists even in the region where all the field vanish" which was discovered by Aharonov and Bohm.

\section{Acknowledgements}

We would like to thank Nam Kim for his help and discussion. This work was supported by a 2-year Research Grant of Pusan National University.

\section{References}

[1] Y. Aharonov and D. Bohm, Phys. Rev. 115, 485 (1959).

[2] A. Tonomura, N. Osakabe, T. Matsuda, T. Kawasaki, and J. Endo,Phys. Rev. Lett. 56, 792 (1986).

[3] S. M. Roy, Phys. Rev. Lett. Phys. Rev. Lett. 44, 111 (1980).

[4] T. H. Boyer, Phys. Rev. D 8, 1679 (1973).

[5] T. H. Boyer, Phys. Rev. A 36, 5083 (1987).

[6] A. Yacoby, M. Heiblum, V. Umansky, H. Shtrikman, and D. Mahalu, Phys. Rev. Lett. 73, 3149 (1994).

[7] A. Yacoby, R. Schuster, and M. Heiblum, Phys. Rev. B 53, 9583 (1996).

[8] D. I. Chang et al., Nature Physics 4, 205 (2008).

[9] D. Chang, H. Lee, and Y. Chung, J. Korean Phys. Soc. 51, 1395 (2007).

[10] Y. Oh, M. Seo, and Y. Chung, J. Korean Vac. Soc. 21, 151 (2012).

[11] I. Thayne et al., Proceedings of 4th IEEE Conference on Nanotechnology (Munich, Germany, August 16-19, 2004), pp. 9597.

[12] B. S. Deaver, Jr. and W. M. Fairbank, Phys. Rev. Lett. 7, 43 (1961).

[13] H. Doll and M. Näbauer, Phys. Rev. Lett. 7, 51 (1961).

[14] T. Morgan-Wall et al., Phys. Rev. Lett. 114, 077002 (2015). 